Article

\title{
Simulation Study on Positive Corona Discharge of Receptors on Rotating Wind Turbine Blade Tips under Thundercloud Electric Fields
}

\author{
$\mathrm{Lu} \mathrm{Qu}^{1}$, Yu Wang ${ }^{2, *}$, Gang Liu ${ }^{1}$, Minchuan Liao ${ }^{1}$, Hansheng Cai ${ }^{1}$, Tao Zhang ${ }^{2}$, Yeqiang Deng ${ }^{2}$ \\ and Xishan Wen ${ }^{2}$ \\ 1 Electric Power Research Institute, China Southern Power Grid, Guangzhou 510080, China; \\ qulu@csg.cn (L.Q.); liugang@csg.cn (G.L.); liaomc@csg.cn (M.L.); caihs@csg.cn (H.C.) \\ 2 School of Electrical Engineering and Automation, Wuhan University, Wuhan 430072, China; \\ zt5040@whu.edu.cn (T.Z.); dengyeqiang@whu.edu.cn (Y.D.); xswen@whu.edu.cn (X.W.) \\ * Correspondence: yuwang@whu.edu.cn
}

Received: 28 October 2019; Accepted: 28 November 2019; Published: 10 December 2019

\begin{abstract}
Recent lightning simulation experiments have not simulated the influence of blade rotation in the long term after corona inception. This study uses a finite element method and considers the adhesion and collision processes of positive ions, aerosol ions, and neutral particles to establish a two-dimensional positive corona discharge model based on a multicomponent diffusion transport equation. The microscopic distribution of these three types of particles and the influence mechanism of charged particles' migration under electric fields and wind were studied. The results show that ion migration is affected by both electric field and wind speed. The higher the wind speed, the larger is the deviation amplitude of charged particles along the direction of wind. With an increase in wind speed, the corona current on the receptor surface gradually increases. When the wind speed is $30 \mathrm{~m} / \mathrm{s}$, the corona current peak value increases by almost six times when compared with that when no wind is present. From this, it can be inferred that blade rotation reduces the concentration of positive ion in the receptor area, thereby facilitating electron avalanche and streamer-leader conversion.
\end{abstract}

Keywords: blade rotation; corona discharge model; finite element approach; receptor

\section{Introduction}

With the rapid increase in the number of wind power installations in China, the capacity of wind power units has been gradually increasing. Therefore, the probability of wind power units being hit by lightning is increasing. According to the data from 2015 from the wind energy equipment branch of the China Association of Agricultural Machinery Manufacturers, the damage rate of wind blades caused by lightning in China's wind fields is as high as $1 \%$, and up to $5 \%$ in some areas with high occurrences of thunderstorms [1]. Blade damage accidents will bring about a huge loss of power, and at the same time generate large maintenance costs [2]. Most wind turbines are rotating when they are struck by lightning. Therefore, the lightning connection and flash discharge characteristics of wind turbines in the rotational state must be studied as a matter of urgency. Wang and Wilson et al. [3-5] have conducted long-term natural observations of lightning contact on wind turbines and have suggested that rotary fans have stronger lightning triggering ability than static towers of the same height. Owing to the small number of effective samples and the lack of comparisons with static fans having the same structure, it has been difficult to support the above inference.

The lightning strike process of wind turbines with rotary blades is similar to that of a tall building under strong wind conditions. Many researchers have studied the effect of wind on discharge. Diaz et al. [6] used a wind tunnel to study the influence of wind speed on the corona current of positive 
and negative discharge. Their results showed that with an increase in wind speed, the corona current peak value for both positive and negative cases increased. In particular, that for positive discharge increased faster. Chaves et al. [7] studied the influence of different needle electrode materials on the corona current generated under windy conditions and found that different materials had similar influences on the corona current of a needle electrode. Based on existing studies on the influence of wind speed on discharge, most researchers have conducted simulation studies through experiments and considered the influence of airflow on corona current under short gaps. However, differences exist between the development process of experimental short gap discharges and an actual lightning strike. To study the lightning discharge process of wind turbines, we designed a 1:30 scale model with an adjustable rotation speed, typical of the typical $2 \mathrm{MW}$ wind turbine the has been examined in previous research [8-11]. We conducted discharge tests with $1 \mathrm{~m}, 2 \mathrm{~m}, 4 \mathrm{~m}$, and $6 \mathrm{~m}$ gaps under the arc electrode, using a -250/2500 $\mu$ s negative switching impulse wave. In the experiment, the arc electrode was made of a steel tube with a diameter of $0.03 \mathrm{~m}$ to simulate the corona diameter of the downward leader head. Two kinds of receptors with typical curvature radii were selected in the experiment, including the receptor cap and the receptor disc. The receptor disc was embedded with an isolated metal point in the blade tip. A circular copper disc with a diameter of $3 \mathrm{~mm}$ was arranged $1 \mathrm{~cm}$ away from the tip of the blade. The receptor cap was the metal conductor layer, used to cover the blade tip completely. Copper foil was used to wrap within $5 \mathrm{~cm}$ of the blade tip. Both of these receptors were connected to the hub with a $3 \mathrm{~mm}$ in diameter copper wire. Our results show that under the conditions of $1 \mathrm{~m}$ and $2 \mathrm{~m}$ gaps, the breakdown voltage between the electrode and blade tip increases with the increase of blade speed. Under the conditions of $4 \mathrm{~m}$ and $6 \mathrm{~m}$ gaps, the breakdown voltage between electrode and blade tip decreases with the increase of blade speed. In the common theories, the rotation of the wind turbine will change the space charge distribution, which will facilitate the initiation of an upward leader [4], so the breakdown voltage will decrease with the increase of blade speed. However, it presently cannot be explained why the effect of rotation speed on the breakdown voltage under different gap conditions is opposite. As such, it is necessary to carry out simulation research to study the discharge development process under the condition of blade rotation.

As for the simulation of the lightning discharge process, numerous researchers have simulated the lightning protection of tall buildings based on the corona model. Aleksandrov et al. [12-14] established a one-dimensional corona model of a hemispherical lightning rod to simulate the diffusion and migration processes of a corona starting from the surface of the lightning rod. The distribution of ions after migration and diffusion was approximately hemispherical. However, simulation with a one-dimensional model involves many approximations, and, therefore, Becerra [15] extended the model from one to two dimensions on the basis of Aleksandrov's research, more accurately analyzing the developmental process of the lightning rod surface corona after its initiation. The simulation results obtained using the two-dimensional model showed that ion migration presents different trends in the horizontal and vertical directions. Compared with the one-dimensional model, the two-dimensional model can reflect the ion migration process more accurately. However, as lightning rods were being investigated, these studies only considered the thundercloud electric field's influence on migration features. Their results did not consider the influence of wind, and they cannot be compared with the corona of the rotary blade receptor under the background of an actual thundercloud. Yanhui Zou [16] established a time domain numerical calculation model of a corona space charge distribution model in DC transmission lines considering wind speed, and the results showed that with the increase of wind speed, the peak value of corona current of ground wires above the negative pole linearly increased. However, there are lots of differences between the process of corona discharge in wires and wind turbine blades, and this result cannot reflect the corona initiation process of an actual wind turbine.

Overall, previous observation studies on wind turbine lightning strikes and the influence of wind speed on discharge have concluded that wind turbine rotation leads to lightning strike characteristics that differ from those of common ground buildings. This requires further discussion. In this study, the COMSOL Multiphysics software was used to establish a corona discharge model of the receptor on the 
tip of a blade under negative thundercloud conditions. The distribution of spatial ions under different wind speeds were studied from the perspective of ion diffusion and migration. The simulation results can be compared with the influence of actual blade rotation on the ion distribution and corona current in the blade tip region under negative thunderclouds, and they can provide a basis for discussing the lightning strike development process under blade rotation.

\section{Numerical Simulation Model Based on Fluid Dynamics}

\subsection{Governing Equation of Fluid Dynamics Numerical Simulation Model}

Under negative thundercloud conditions, when the receptor surface reaches the initial corona field intensity, corona discharge will occur. Corona discharge will ionize to produce electrons and positive ions, electrons will quickly enter the receptor, and positive ions will migrate toward the thundercloud. In the simulation, the process of electron collapse in space was not considered. To bring the simulation results closer to the development process after actual corona initiation, the process of positive ions constantly adhering to and colliding with other ions and then reacting to form other charged particles during the generation and outward diffusion of positive ions was fully considered in this model. Accordingly, this model considers three types of particles in the background space of thundercloud, namely, positive ions, $n_{+}$, aerosol ions, $N_{+}$, and neutral particles, $N_{a}$. Physical processes such as adhesion and collision between the three types of particles can be expressed by multicomponent transport equations which are coupled with each other, which are shown as follows [15].

$$
\begin{gathered}
\frac{\partial n_{+}}{\partial t}=D \nabla^{2} n_{+}-\nabla \cdot\left(n_{+}\left(\mu_{n_{+}} \vec{E}+\vec{W}\right)\right)-k_{n N} n_{+} N_{a} \\
\frac{\partial N_{+}}{\partial t}=D \nabla^{2} N_{+}-\nabla \cdot\left(N_{+}\left(\mu_{N_{+}} \vec{E}+\vec{W}\right)\right)+k_{n N} n_{+} N_{a} \\
\frac{\partial N_{a}}{\partial t}=D \nabla^{2} N_{a}-k_{n N} n_{+} N_{a}
\end{gathered}
$$

Here, $\mu_{n+}\left[1.5 \times 10^{-4} \mathrm{~m}^{2} /(\mathrm{V} \cdot \mathrm{s})\right]$ is the electrical mobility of positive ions, $\mu_{N+}\left[1.5 \times 10^{-4} \mathrm{~m}^{2 /}(\mathrm{V} \cdot \mathrm{s})\right]$ is the electrical mobility of aerosol ions, $W$ is the wind speed, set as going horizontally to the right on the vertical plane and set to different values depending on the requirements, $k_{n N}\left(2.9 \times 10^{-12} \mathrm{~m}^{3} / \mathrm{s}\right)$ is the adhesion coefficient, and $D\left(1 \mathrm{~m}^{2} / \mathrm{s}\right)$ is the diffusion coefficient. Furthermore, $e$ and $\varepsilon_{0}$ denote the elementary charge and vacuum dielectric constant, respectively [17].

In addition, the electric field distribution in the ionization region can be expressed by the electric field Poisson equation:

$$
\nabla \cdot \vec{E}=-\nabla^{2} \phi=\frac{e\left(n_{+}+N_{+}\right)}{\varepsilon_{0}}
$$

By normalizing the above equation and solving it using discrete numerical differences, the ion migration characteristics under thunderclouds and different wind speeds can be solved.

This model must consider corona characteristics under negative thunderclouds as well as the migration of charged particles influenced by the electric field and wind. Therefore, the electrostatic module and convection/diffusion module in COMSOL Multiphysics were used to simulate the influence of wind speed on ion migration through mutual coupling between the two modules. The transient solver PARDISO in COMSOL Multiphysics was used to solve the problem, and the time stepping method was implicit. If the explicit scheme is adopted, the time step shall meet the Courant stability condition, that is, the selection of the time step must ensure that the transfer speed of the node charge density information is less than the charge transfer speed, otherwise the solution of the particle transport equation will diverge with time, and the number of calculation time steps will be very large, which makes the calculation time difficult to accept. Therefore, this paper adopts implicit discrete equations to overcome this problem and improve the computational efficiency [16]. Here, the time step used is $(0,0.2,25)$ and the relative tolerance is 0.001 . 


\subsection{Basic Parameters and Geometric Model Settings}

In a thundercloud, the background electric field, $E_{0}$, keeps increasing within a certain duration $\tau$. After it reaches the maximum value, $E_{0 \max }$, the thundercloud electric field remains unchanged until it decreases sharply after a lightning strike. The simulation in this paper focus on the initial stage of the corona on the wind turbine blade before a lightning strike. Researchers have noted that an electric field can be expressed by a piecewise function that increases linearly within time $\tau$, where $E_{0 \text { max }}$ remains unchanged until a lightning strike occurs $[18,19]$. The background electric field $E_{0}$ is expressed as follows:

$$
E_{0}=\left\{\begin{array}{cc}
(t / \tau) E_{0 \max } & t<\tau \\
E_{0 \max } & t \geq \tau
\end{array}\right.
$$

Extensive measurements have shown that about $90 \%$ of cloud-to-ground lighting strikes are of the negative lightning type, so the study of lightning is mainly focused on negative polarity. Consequently, the thundercloud electric field was set to a negative polarity.

In the simulation considering the influence of wind speed on ion migration, the receptor is equivalent to a cylindrical model with ground clearance height $h$ and bottom surface radius $r_{0}$. To simplify the simulation calculation, a section parallel to the base of the cylinder was made and the three-dimensional model of the flash connector was simplified into a two-dimensional model in the $x-y$ plane. The receptor can be equivalent to a circle on the $x-y$ plane. Figure 1 shows the geometric model and mesh subdivision.

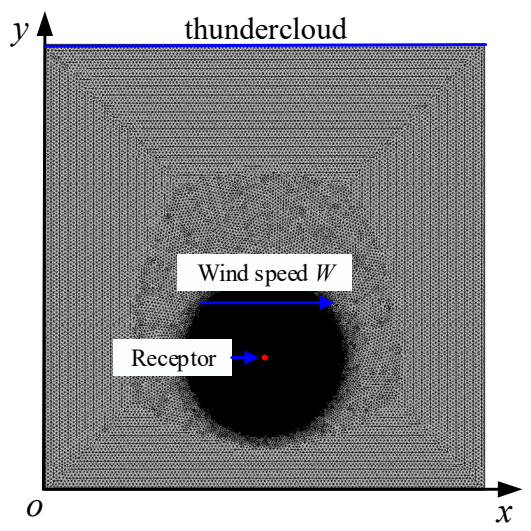

Figure 1. Geometric model and mesh generation.

To reduce the number of subdivision cells and the calculation time, the height $h$ was set to $15 \mathrm{~m}$, the receptor radius $r_{0}$ was set to $0.5 \mathrm{~cm}$, the maximum electric field of thundercloud $E_{0 \mathrm{max}}$ was set to $40 \mathrm{kV} / \mathrm{m}$, and the lift time $\tau$ was set to $20 \mathrm{~s}$ [20].

As the computational model mainly focuses on the electrical parameters of the space near the receptor, considering the large gap between the receptor and the geometric size of the whole computational space, the grid density of the receptor and the space nearby should be increased during the generation. Making concentric circles with the center of the receptor as the center of the circle with radii of $50 \mathrm{~cm}$ and $500 \mathrm{~cm}$ respectively, the outermost boundary is a square with a length of $5000 \mathrm{~cm}$ and the grid size increases successively from the receptor outward. Mesh generation of the model adopts free mesh generation for a triangular mesh and the minimum unit size is shown in Table 1.

Under the action of the thundercloud electric field, the receptor surface induces an electric field that will gradually increase with the background electric field. When it reaches the initial corona field $E_{i}$, the receptor surface starts to generate positive ions that spread outward from the surface. The corona initial field strength can be calculated using Peek's formula [21]:

$$
E_{i}=3.1\left(1+0.0308 r_{0}^{-1 / 2}\right)
$$


Table 1. The minimum size of mesh subdivision.

\begin{tabular}{cc}
\hline Area & Minimum Unit Size \\
\hline Receptor & $0.1 \mathrm{~cm}$ \\
First circle & $0.375 \mathrm{~cm}$ \\
Second circle & $0.625 \mathrm{~cm}$ \\
Square & $1.5 \mathrm{~cm}$ \\
\hline
\end{tabular}

\subsection{Setting of Boundary Conditions and Simulation Parameters}

In the simulation calculations, the boundary conditions in each module must be set. The modules selected for this simulation were the electrostatic and convection/diffusion modules. The boundary conditions were set as follows.

In the electrostatic module, the background potential value $V=-E_{0} H$ was applied at the upper boundary. It should be ensured that the entire simulation area is in the background electric field of thundercloud $E_{0}$. The left and right boundaries were set to zero charge, and the receptor and lower boundary were set to ground.

In the convection/diffusion module, the outer boundary was set to zero flux and the concentration boundary of positive ion $n_{+}$was set on the receptor surface to ensure that the generated positive ions could always maintain the motion field intensity $E$ on the receptor surface after the corona started [12-14,22]. In the simulation calculations, the initial concentrations of the pace positive ion $n_{+}$ and aerosol ion $N_{+}$were set to 0 , and that of the neutral particle $N_{a}$ was set to $10^{11} / \mathrm{m}^{3}$ [12].

\section{Simulation Results}

The spatiotemporal distribution of positive particles, aerosol particles, and neutral particles under different wind speeds were simulated according to the 2D corona simulation model of the receptor under negative thunderclouds.

\subsection{Influence of Wind Speed on the Spatiotemporal Distribution of Positive Ions}

\subsubsection{Positive Ion Distribution When Wind Speed is 0}

To study the law of positive ion migration under the thundercloud electric field, a contour map of the positive ions' spatial density distribution at different times when the wind speed is zero was drawn, as shown in Figure 2.

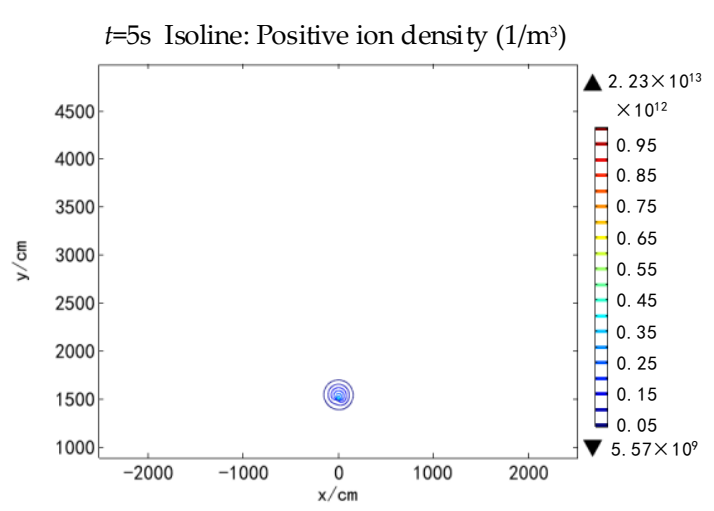

(a)

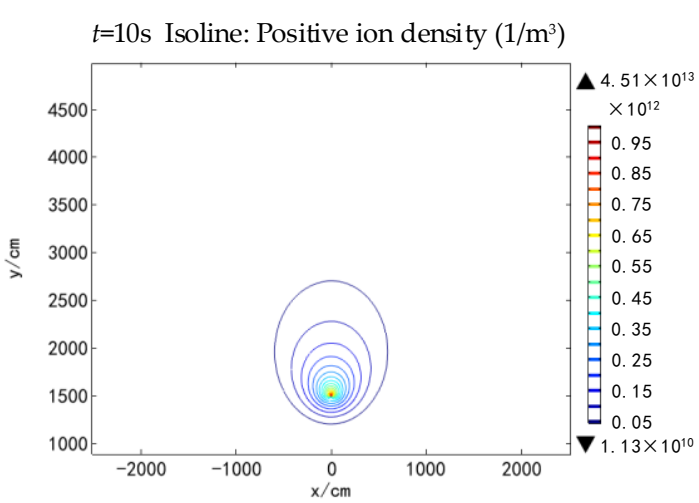

(b)

Figure 2. Cont. 


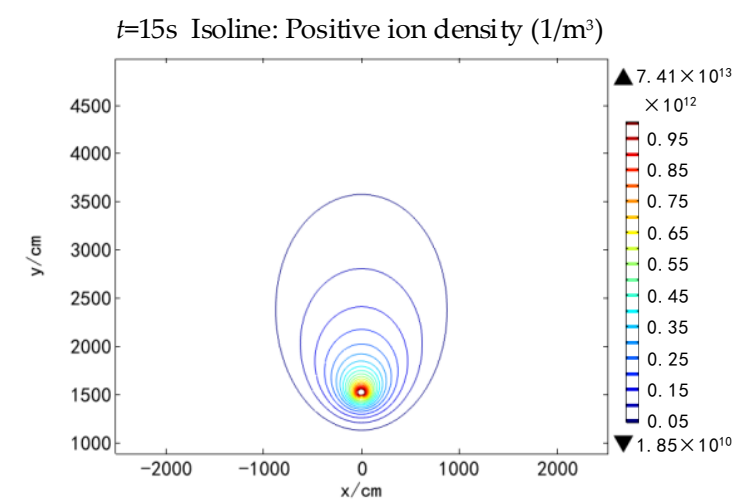

(c)

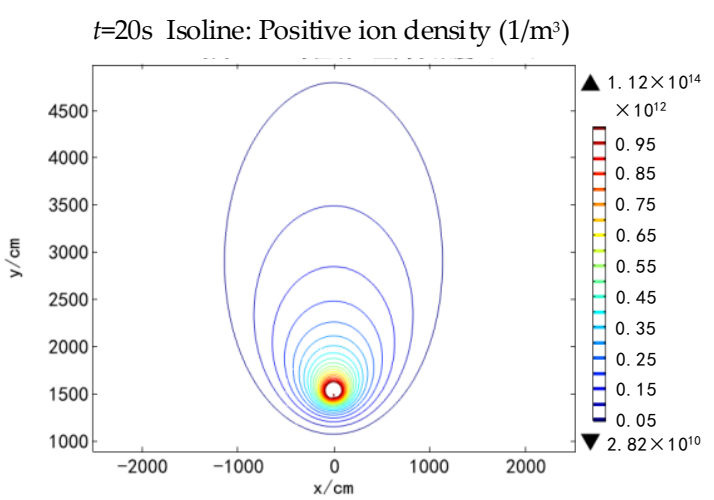

(d)

Figure 2. Spatial isolines of positive ion density at different moments when the wind speed was 0 .

(a) $t=5 \mathrm{~s}$; (b) $t=10 \mathrm{~s}$; (c) $t=15 \mathrm{~s}$; (d) $t=20 \mathrm{~s}$.

This map shows that when the surface corona starts on the receptor, positive ions near the receptor are evenly distributed and those in the upper and lower parts of the flash connector are approximately symmetrical. This is due to the presence of a large amount of space charge in the area near the receptor; this charge counteracts some of the effect of the thundercloud electric field. As time passes, the corona continues to develop, where ions migrate further outward and the space charge interaction gradually weakens. Then, the thundercloud electric field becomes the main factor affecting ion migration. Most positive ions migrate vertically to the ground, and their density isolines gradually show an elliptical distribution.

\subsubsection{Influence of Different Wind Speeds on Positive Ion Migration}

To study the influence of wind speed on the diffusion and migration of positive ions, at $t=20 \mathrm{~s}$, the contour map of the positive ions' spatial density distribution at $W=0,10,20$, and $30 \mathrm{~m} / \mathrm{s}$ was drawn, as shown in Figure 3.

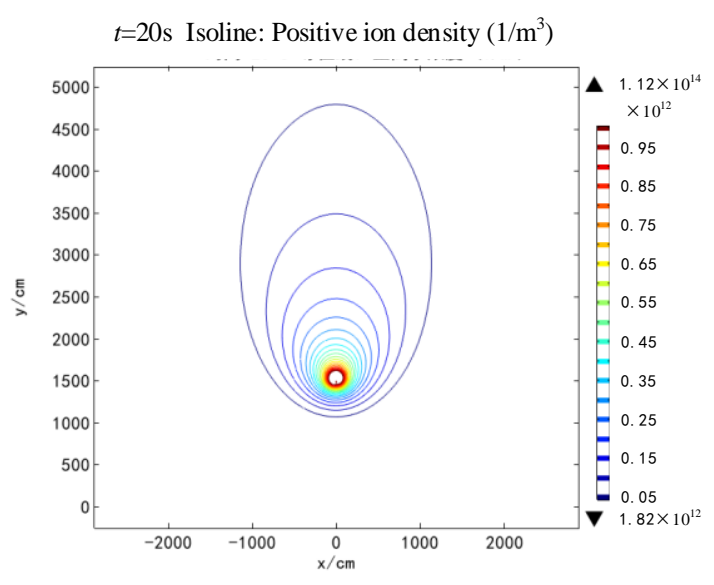

(a)

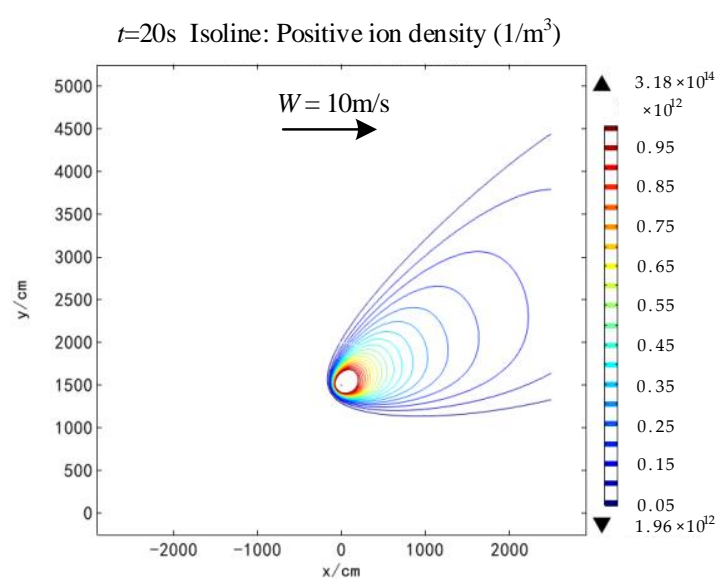

(b)

Figure 3. Cont. 


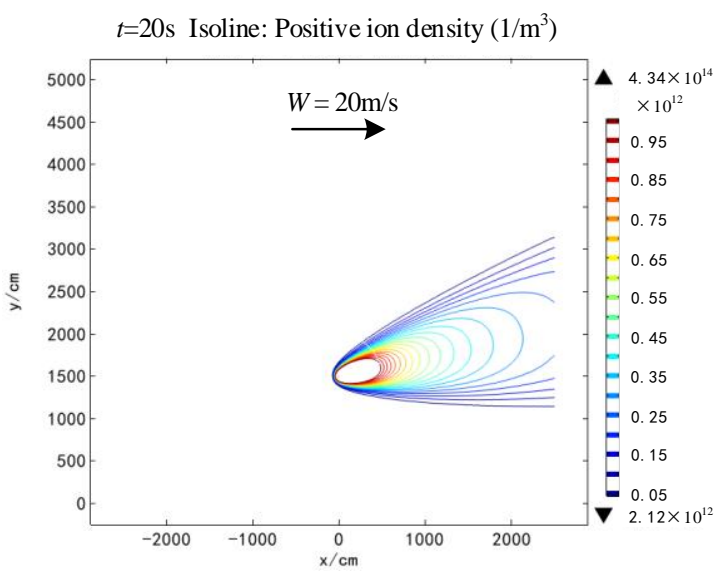

(c)

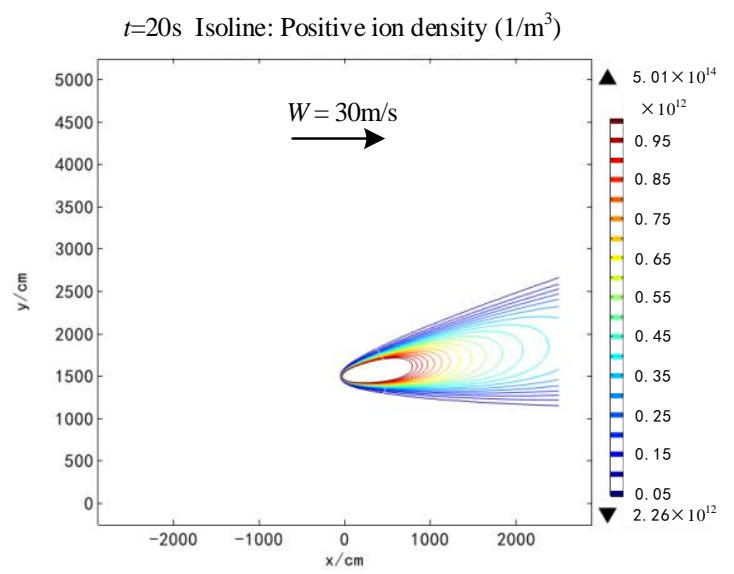

(d)

Figure 3. Isopleth spatial distribution of positive ion density at different wind speeds when $t=20 \mathrm{~s}$. (a) $W=0 ;$ (b) $W=10 \mathrm{~m} / \mathrm{s} ;$ (c) $W=20 \mathrm{~m} / \mathrm{s} ;$ (d) $W=30 \mathrm{~m} / \mathrm{s}$.

This map shows that differences exist in the migration and diffusion characteristics of positive ions under different wind speeds. The ion migration process is affected by both the electric field force and wind. In the area close to the receptor, ion migration is mainly affected by the induced electric field of the surface charge and corona charge of the receptor, and the ion distribution remains relatively uniform. As the ions spread outward from the receptor, the background electric field causes most ions to migrate toward the thundercloud, whereas horizontal wind causes particles to drift to the right during migration. Therefore, when there is wind in the space background, the ions present an elliptical distribution state with rightward deviation in the plane of the receptor, and the larger the wind speed, the larger the deviation amplitude.

We took the transversal $X$ of the $x$-axis when $x=0$ and drew a distribution diagram of positive ions at different times under different wind speed conditions along the transversal $X$, as shown in Figure 4.

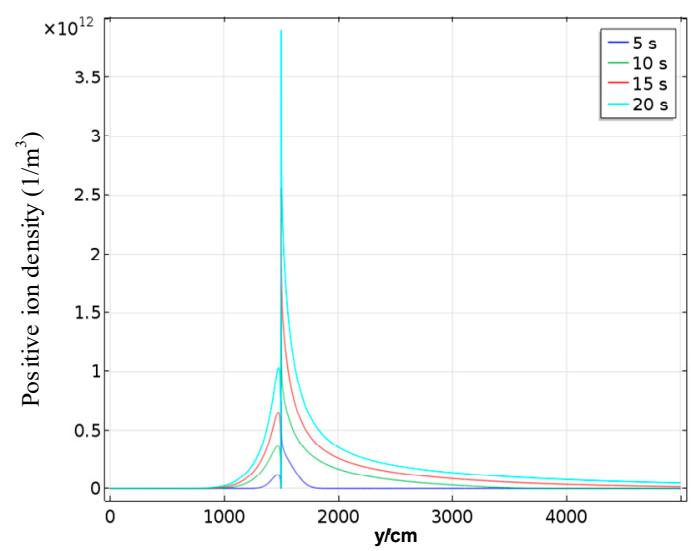

(a)

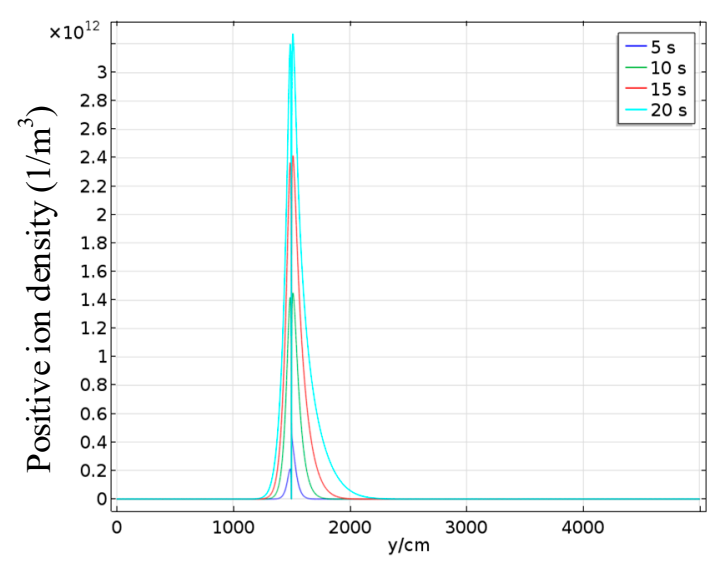

(b)

Figure 4. Cont. 


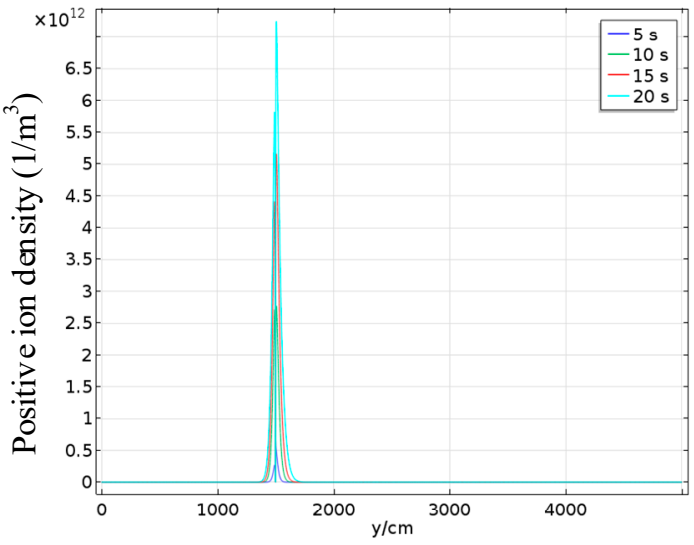

(c)

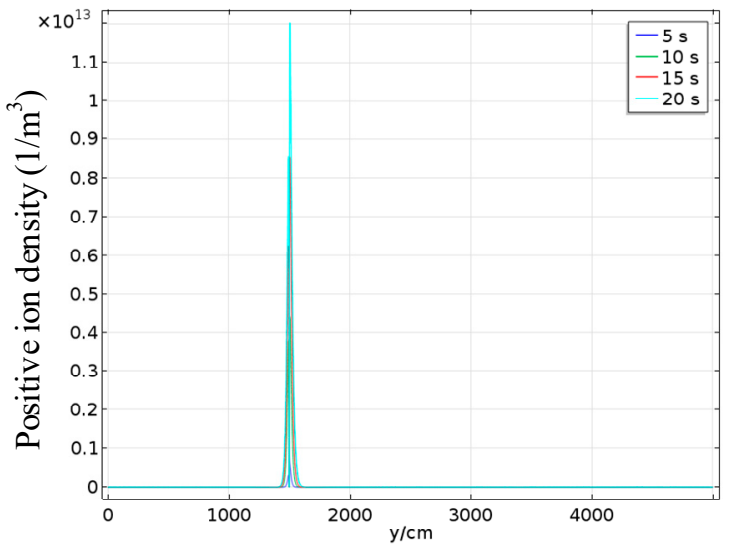

(d)

Figure 4. Distribution of positive ion density at each moment on the transversal $X$ under different wind speed conditions. (a) $W=0 ;$ (b) $W=10 \mathrm{~m} / \mathrm{s} ;$ (c) $W=20 \mathrm{~m} / \mathrm{s}$; (d) $W=30 \mathrm{~m} / \mathrm{s}$.

Under different wind speed conditions, positive ions were mainly concentrated in the area near the receptor, and their concentration decreased when moving further away from the receptor. Under different wind speeds, the decay speed of positive ion density along the y-axis was different. The higher the wind speed, the faster the decay speed of positive ion density along the $y$-axis. For $t=20 \mathrm{~s}$, when the wind speed was 0 , the concentration of positive ions migrating upward and downward along the y-axis tended to 0 near $y=10 \mathrm{~m}$ and $y=45 \mathrm{~m}$, respectively. For a wind speed of $30 \mathrm{~m} / \mathrm{s}$, positive ions distributed along the $y$-axis mostly existed in the area between $y=14 \mathrm{~m}$ and $y=16 \mathrm{~m}$. This proved that wind had an important influence on the spatial positive ion migration.

To further analyze the influence of wind on ion migration, the transversal $X$ was divided into the upper $\left(X_{\text {up }}\right)$ and lower transversals $\left(X_{\text {down }}\right)$ with a cut-off point at $y=15 \mathrm{~m}$, and the line integral of the charge density on the two sections of the line was obtained along $X_{\text {up }}$ and $X_{\text {down }}$. Table 2 shows the calculation results for charges $Q_{\mathrm{up}}$ and $Q_{\text {down }}$ and the total charge $\left(Q_{\mathrm{all}}\right)$ on transversal $X$.

Table 2. Calculation results of the charge Amount on transversal $X$ under different wind speed conditions at different times.

\begin{tabular}{cccccc}
\hline$W(\mathbf{m} / \mathbf{s})$ & $\boldsymbol{T}(\mathbf{s})$ & $Q_{\text {up }}(\mathbf{m u ~ C} / \mathbf{m})$ & $Q_{\text {down }}(\mathbf{m u ~ C} / \mathbf{m})$ & $Q_{\text {all }}(\mathbf{m u ~ C} / \mathbf{m})$ & $Q_{\text {up }} / Q_{\text {down }}$ \\
\hline \multirow{2}{*}{0} & 10 & 0.436 & 0.098 & 0.534 & 4.45 \\
& 15 & 0.828 & 0.172 & 1.000 & 4.81 \\
& 20 & 1.263 & 0.262 & 1.525 & 4.82 \\
\hline \multirow{2}{*}{10} & 10 & 0.177 & 0.120 & 0.297 & 1.48 \\
& 15 & 0.394 & 0.230 & 0.694 & 1.71 \\
& 20 & 0.683 & 0.347 & 1.030 & 1.97 \\
\hline \multirow{2}{*}{20} & 10 & 0.144 & 0.117 & 0.261 & 1.23 \\
& 15 & 0.317 & 0.216 & 0.533 & 1.47 \\
& 20 & 0.528 & 0.320 & 0.848 & 1.65 \\
\hline \multirow{2}{*}{30} & 10 & 0.141 & 0.110 & 0.251 & 1.28 \\
& 15 & 0.305 & 0.200 & 0.505 & 1.53 \\
& 20 & 0.490 & 0.300 & 0.790 & 1.63 \\
\hline
\end{tabular}

Table 2 shows that when wind speed is constant, $Q_{\text {down }}$ is smaller than $Q_{\text {up }}$, and the proportion of $Q_{\text {up }}$ in $Q_{\text {all }}$ increases. Under the thundercloud electric field, most positive ions move toward the thundercloud above the receptor.

In addition, according to the charge distribution law under different wind speeds, as listed in Table 2, both $Q_{\text {up }}$ and $Q_{\text {down }}$ simultaneously decreased with an increase in wind speed. For $W=0 \mathrm{~m} / \mathrm{s}$, 
$Q_{\text {all }}$ is around half that in the case of no wind. This is because as wind speed increases, rightward positive ion migration gradually increases and the charge on transversal $X$ gradually decreases. The aforementioned charge distribution law indicates that the presence of wind affects positive ion migration in space.

\subsection{Influence of Wind Speed on Corona Current Variation Rule}

To study the influence of wind speed on the variation trend of the corona current on the receptor surface, the corona current curve over time at $W=0,10,20$, and $30 \mathrm{~m} / \mathrm{s}$ was drawn according to Equation (7), shown in Figure 5. The corona current on the receptor surface can be expressed as follows:

$$
I(t)=2 \pi r_{0} e n_{+}\left(r_{0}, t\right) \mu_{n+} E_{i}
$$

where $n\left(r_{0}, t\right)$ is the positive ion density on the receptor surface as obtained through the simulation.



Figure 5. Variation of corona current on the surface of the receptor under different wind speeds.

The corona current on the receptor surface exhibits the same trend under different wind speeds. As described in Section 2.2, the thundercloud background electric field keeps increasing within a certain duration, namely, $\tau(\tau=20 \mathrm{~s})$. The corona current started from the time when the corona appeared on the receptor surface $(t=4.6 \mathrm{~s})$. For $t<20 \mathrm{~s}$, the corona current increased with time as the thundercloud electric field gradually increased. The corona current peaked at $t=20 \mathrm{~s}$. For $t>20 \mathrm{~s}$, the thundercloud electric field stopped increasing and the corona current amplitude decreased slightly and then stabilized. The simulation results of the corona current obtained in this section for $W=0$ were compared with the corona current of a $60 \mathrm{~m}$ tall rod with a $0.02 \mathrm{~m}$ cap radius, computed by means of Becerra's corona model [11], in which the corona current of a $1 \mathrm{~m}$-tall pencil-shaped rod computed with the 2D model was compared with the measured result in [23] to verify the accuracy of the model. The corona current variation trend of the two models were found to be consistent, and the order of magnitude were both in the $0.1 \mathrm{~mA}$ range, thus verifying the correctness of the proposed model.

\subsection{Influence of Blade Rotation on Positive Upward Leader Initiation}

As stated, a charged particle distribution model of the receptor on the blade tip, considering the wind speed and background of a negative thundercloud, has been established. The simulation results have revealed that positive ions diffuse outward in the direction of the electric field after the corona starts. In the initial stage, the positive ions were evenly distributed in the upper and lower regions of the receptor. However, as positive ions gradually moved away from the receptor, they migrated and spread toward the direction of the thundercloud. Because the migration speed of electrons is 
approximately two orders of magnitude higher than that of positive ions, newly ionized electrons will quickly enter the receptor, resulting in the formation of a positive ion space area above the receptor. The decrease in field intensity in the positive ion space region inhibits electron collapse near the receptor and impedes streamer development. Simultaneously, the existence of positive ions promotes an increase in the collision surface of the gas and increases the probability of electron recombination. These two factors jointly inhibit electron collapse and further streamer development, inhibiting the generation of the upward leader.

Simultaneously, the wind speed has an effect on the migration and diffusion of space ions. Under windy conditions, the charged particles deviate in the direction of wind. The higher the wind speed, the larger the deviation. Positive ions present an elliptical distribution with a rightward deviation, and this weakens the positive ion density in the area directly above the receptor. Simultaneously, the corona current increases with an increase in wind speed, indicating that the corona current flowing through the receptor surface has increased. Electron avalanche in the streamer area developed more violently in this case. This was more conducive to streamer development. Therefore, when the blade rotates, it is equivalent to the presence of wind around the receptor, and this changes the positive ion distribution nearby and facilitates the formation of the upward leader.

The variation of breakdown voltage with blade rotation in our previous experiment [8-11] can be explained as follows, where the experimental gap of 1-6 $\mathrm{m}$ is quite different from the actual height of the thundercloud. With such a gap length, the positive ion space area above the receptor not only weakens the upward leader on the surface of receptor, but also increases the field intensity between the area and the high-voltage electrode, which is conducive to the formation of secondary electron avalanche in the external area and promotes the upward leader to extend to the high-voltage electrode. When the gap is 1-2 $\mathrm{m}$, the positive ion space area is close to the high-voltage electrode, and its promotion plays a major role in the upward leader development. Therefore, with the increase of wind speed, the positive ion offset increases and the breakdown voltage increases. When the gap length increases to 4-6 m, the positive ion space area has a weaker promoting effect on the upward leader development, and its weakened effect is dominant, so the breakdown voltage decreases with the increase of wind speed.

\section{Conclusions}

This paper focuses on the corona discharge process and explains the abnormal phenomena in the experiment. By using the COMSOL Multiphysics finite element software, this study established a distribution model of charged ions at the receptor on the blade tip, considering the wind speed and electric field strength of thunderclouds. The attachment and collision processes of positive ions, aerosol ions, and neutral particles were considered in the model. The model can reflect the migration and spatial distribution of these three types of particles on the receptor surface under thunderclouds. The main conclusions are as follows:

(1) Wind speed has an effect on the migration and diffusion of space ions. Under windy conditions, ion migration is affected by both the electric field force and wind. In the area near the receptor, ion migration is mainly affected by the thundercloud and the electric field induced by the electric charge, and the ion distribution is relatively uniform. As the charged particles gradually spread away from the receptor, the field intensity decreases, and the influence of wind speed on the particle distribution gradually increases. Charged particles are offset in the direction of the wind. The higher the wind speed, the larger the deviation amplitude.

(2) Under the action of an electric field, when the surface field intensity of the receptor reaches the initial corona field intensity, positive ions are generated on the receptor. As a result, positive ions gradually accumulate in the area near the receptor and a positive space charge region is formed, thereby inhibiting the movement of electrons and reducing the probability of an electron avalanche developing in the streamer. Under the action of wind, the positive charge space region is offset, and the concentration is reduced. This is conducive to electron collapse and the streamer-leader conversion process. 
(3) Blade rotation weakens the accumulation of positive ions in the blade tip area. This is conducive to the generation and development of the upward leader and makes the wind turbine more vulnerable to lightning strikes. This study represents basic research on wind turbine lightning protection and provides some related reference calculation values.

Future research should focus on extending the simulation process to the whole process of long gap discharge, and then to the discharge process of natural lightning, so as to guide the transformation of lightning protection through the law of lightning simulation calculation.

Author Contributions: Conceptualization, H.C., L.Q. and Y.W.; Methodology, L.Q. and Y.W.; Software, L.Q.; Validation, G.L. and Y.D.; Formal Analysis, L.Q. and Y.W.; Investigation, M.L.; Writing-Original Draft Preparation, L.Q. and T.Z.; Writing-Review \& Editing, T.Z. and X.W.; Supervision, H.C.; Project Administration, Y.W.

Funding: This research received no external funding.

Conflicts of Interest: The authors declare no conflict of interest.

\section{References}

1. Li, Q.; Guo, Z.; Zhang, L.; Siew Wah, H. Key Issues with Lightning Protection Research of the Large-scale Wind Farms. Proc. CSEE 2018, 38, 5296-5306.

2. IEC 61400-24. 2002 Wind Turbine Generator Systems_Part 24: Lightning Protection; IEC: London, UK, 2002.

3. Wang, D.; Takagi, N.; Watanabe, T.; Sakurano, H.; Hashimoto, M. Observed characteristics of upward leaders that are initiated from a windmill and its lightning protection tower. Geophys. Res. Lett. 2008, 35, 1-5. [CrossRef]

4. Wang, D.; Takagi, N. Characteristics of Winter Lightning that Occurred on a Windmill and Its Lightning Protection Tower in Japan. IEEJ Trans. Power Energy 2012, 132, 568-572. [CrossRef]

5. Nicholas, W.; Jackson, M.; Cummins, D.K. Lightning Attachment to Wind Turbines in Central Kansas: Video Observations, Correlation with the NLDN and In Situ Peak Current Measurements; The European Wind Energy Association: Vienna, Austria, 2013.

6. Jorge, D.; Gustavo, O.; Sebastian, C.; Román, F. Positive and Negative Point Discharge Corona Currents: Influence of Thunderstorm-like Wind Velocities. In Proceedings of the 2014 International Conference on Lightning Protection (ICLP), Shanghai, China, 11-18 October 2014.

7. Sebastián, C.; Gustavo, O.; Jorge, D. Measurement of the Corona Space Charge in a wind tunnel. In Proceedings of the 2013 International Symposium on Lightning Protection (XII SIPDA), Belo Horizonte, Brazil, 7-11 October 2013.

8. Wang, Y.; Qu, L.; Si, T.; Ni, Y.; Xu, J.; Wen, X. Experimental study of rotating wind turbine breakdown characteristics in large scale air gaps. Plasma Sci. Technol. 2017, 19, 108-115. [CrossRef]

9. Wang, Y.; Qu, L.; Si, T.; Ni, Y.; Xu, J.; Wen, X. Experimental Study of the Influence of the Blade Rotation on Triggered Lightning Ability of Wind Turbine's Blades. Proc. CSEE 2017, 137, 2151-2158.

10. Li, L.; Si, T.; Wang, Y.; Qu, L.; Xu, J.; Wen, X. Experimental Study on Lightning Discharge Characteristic of Wind Turbine's Rotating Blades. Power Syst. Technol. 2018, 42, 1328-1334.

11. Qu, L.; Wang, Y.; Si, T.; Wen, X. Experimental Study on the Influence of the Air Terminal on Triggered Lightning Ability of Rotation Wind Turbine. High Volt. Eng. 2017, 43, 1628-1634.

12. Aleksandrov, N.L.; Bazelyan, E.M.; Carpenter, R.B.; Drabkin, M.M.; Raizer, Y.P. The effect of coronae on leader initiation and development under thunderstorm conditions and in long air gaps. J. Phys. D Appl. Phys. 2001, 34, 3256-3266. [CrossRef]

13. Aleksandrov, N.L.; Bazelyan, E.M.; Drabkin, M.M.; Carpenter, R.B., Jr.; Raizer, Y.P. Corona Discharge at the Tip of a Tall Object in the Electric Field of a Thundercloud. Plasma Phys. Rep. 2002, 28, 1032-1045. [CrossRef]

14. Bazelyan, E.M.; Raizer, Y.P.; Aleksandrov, N.L. Corona processes and lightning attachment: The effect of wind during thunderstorms. Atmos. Res. 2009, 94, 436-447. [CrossRef]

15. Becerra, M. Glow corona generation and streamer inception at the tip of grounded objects during thunderstorms: Revisited. J. Phys. D Appl. Phys. 2013, 46, 135205-135219. [CrossRef]

16. Zou, Y. Simulation Research on the Effect of Corona Space Charge on the Lightning Shielding Performance of HVDC Transmission Lines. Master's Dissertation, Huazhong University of Science \& Technology, Wuhan, China, 2015. 
17. Qie, X.; Soula, S.; Chauzy, S. Influence of ion attachment on the vertical distribution of the electric field and charge density below a thunderstorm. Ann. Geophys. 1994, 12, 1218-1228. [CrossRef]

18. Wang, Y.; An, Y.; Wen, X.; Li, Z.; Lin, L.; Dai, M.; Gu, D. Research on Typical Long Air Gaps with Negative Switching Impulses (III)-Statistical Character. Proc. CSEE 2014, 46, 6530-6535.

19. Gopalakrishnan, V.; Pawar, S.D.; Murugavel, P. Electrical characteristics of thunderstorms in the Eastern part of India. J. Atmos. Sol. -Terr. Phys. 2011, 73, 1876-1882. [CrossRef]

20. Shuochao, F. Research on the Ultra Corona Characteristics of Thin Wire and Its Application on the Lightning Protection in EHV Transmission Lines. Ph.D. Dissertation, Chongqing University, Chongqing, China, 2015.

21. Peek, F.W. Dielectric Phenomena in High Voltage Engineering; Mc-Graw-Hill: New York, NY, USA, 1929.

22. Bazelyan, E.M.; Raizer, Y.P.; Aleksandrov, N.L. Corona initiated from grounded objects under thunderstorm conditions and its influence on lightning attachment. Plasma Sources Sci. Technol. 2008, 17. [CrossRef]

23. Alessandro, D.; Berger, G. Laboratory studies of corona emissions from air terminals. J. Phys. D Appl. Phys. 1999, 32, 2785-2790. [CrossRef]

(C) 2019 by the authors. Licensee MDPI, Basel, Switzerland. This article is an open access article distributed under the terms and conditions of the Creative Commons Attribution (CC BY) license (http://creativecommons.org/licenses/by/4.0/). 